\title{
A Review of Task Deployment in Cloud Environment for Load Balancing using Unsupervised Learning
}

\author{
Saifali Hanwat \\ Dept. of Computer Science \& Engineering \\ Samrat Ashok Technological Institute \\ Vidisha \\ India
}

\author{
Vivek Sharma \\ Asst. Prof \\ Dept. of Computer Science \& Engineering \\ Samrat Ashok Technologica \\ Institute Vidisha India
}

\begin{abstract}
The task deployment and load balancing is major issue in cloud environments. The deployment of task in cloud environments used data centers. The physical host of data center overloaded due to unexpected transfer of task in environments. Due to in overloading of physical host the processing of data centers is overloaded and the efficiency of cloud environment is decreases. In this paper presents the review of task deployment and load balancing technique used different unsupervised learning algorithm for the balancing of task in cloud environments. The unsupervised learning gives the variety of algorithm such as clustering and some probability based function for the grouping of data and task. In current decade, various cloud scholar used clustering technique for the balancing of load. The clustering technique separates the cloud load in different scenario and load are distributed over other physical component of cloud environments.
\end{abstract}

\section{Keywords}

Cloud Computing, Load Balancing, Data Centers. Machine Learning.

\section{INTRODUCTION}

Cloud computing is service bucket of diversity over the internet. The diversity of bucket influence the various problem in terms of load balancing in terms of task deployments and physical host of data centers. The physical host of data centers is overloaded the performance of cloud environment is degraded. For deployment of task and balancing of load used various algorithm and model [1]. The machine learning provides various algorithm for the balancing of load. The clustering technique is very effective and applicable technique used in dynamic load balancing technique. in dynamic load balancing technique used the partition clustering technique for the partition of load in distributed manners [2]. The dynamic load balancing technique adopt the heuristic and meta heuristic functions for the balancing of load in cloud environments. The meta heuristic function such as particle swarm optimization and ant colony optimization algorithm is used in many variants $[3,4]$. The variants of functions balance the load but faced a problem of certain limitation. In the journey of balancing used hybrid and populistic models for the balancing of load over the cloud computing. Task scheduling is done after the resources are allocated to all cloud entities. Scheduling defines the way different entities are provisioned. Resource provisioning defines which resource will be available to meet user requirements whereas task scheduling defines the way the allocated resource is available to the end user. Task scheduling provides "Multiprogramming Capabilities" in cloud computing environment $[5,6,7]$.The objective of load adjusting is to successfully disperse the work stack between accessible assets, to amplify the advantage from those assets and to have brisk registering and preparing for the customer demands. It is done to make asset use successful and to enhance the reaction time of the employment, at the same time evacuating a condition in which a portion of the hubs are over stacked while some others are under stacked. To assess the nature of a heap adjusting strategy, design or framework, numerous measurements can be utilized. Some of them must be amplified while others ought to be limited, keeping in mind the end goal to have a proficient load adjusting framework. Dynamic grouping is one of the calculation uses to improve the execution of load dispersed to the hubs. The beginning behind the dynamic bunching comparable hubs is assembled together called go between

\section{RELATED WORK}

In this section discuss the related work in the field of load balancing in cloud computing. For the balancing of load in cloud environments used various technique and method based on optimization algorithm, clustering technique and combination of both.

Jia Zhao, Kun Yang, Xiaohui Wei, Yan Ding, Liang Hu, and Gaochao Xu Et al. [1] They talked about, LB-BC has consolidated likelihood hypothesis and the bunching thought to choose the ideal hosts set, where these physical hosts have the most residual figuring power as of now, to deploy and executing errands by selecting the physical host with the greatest posteriori likelihood esteem as the grouping focus and in this way to accomplish the heap adjusting impact from the long haul point of view. Recreation tests show that the examined LB-BC approach can send the moment undertakings rapidly and successfully in cloud server farms. It makes cloud server farms accomplish a long-haul stack adjusting of the entire system.

Ariharan V and Sheeja S Manakattu Et al. [2] They present neighbor mindfulness and forecast instruments to additionally enhance the choice procedure of hubs for irregular walk. The examined calculation chooses the slightest stacked hub from the neighbor list for the irregular walk. From the reproduction, unmistakably selecting slightest stacked hub for irregular walk performs superior to likelihood based choice or the enhanced arbitrary walk calculation. Over an expansive thick system, minimum stacked neighbor of one hub may get stacked by some different hubs and turn out to be intensely stacked hub.

Catalin-Constantin Usurelu, Mihaela-Catalina Nita, Roxana Istrate, Florin Pop and Nicolae Tapus Et al. [3] They actualized a test system for the datacenter and the bug work 
overlay, the reproduced assets being examined from a Gaussian dispersion. As per specialist, every hub of the overlay is equipped for offering the best limit with regards to a specific asset while offering littler capacities with regards to alternate assets. Reproduction results are empowering as their techniques achieve a general coordinated load between half in the most pessimistic scenario and $85 \%$ in the best case. Both techniques demonstrate stable server load and overlay topological solidness, proposing that versatile server provisioning won't not be required as the system keeps up its frame over the term of their analyses.

Naidila Sadashiv and S. M Dilip Kumar Et al. [7] They have introduced a point by point examination on the three figuring models, group, matrix and distributed computing. The issues and difficulties identified with these registering models are highlighted. The undertakings and applications in different fields are quickly examined. Such a correlation in alternate points of view will make straightforward the registering models since the elements of these processing models is by all accounts comparative theoretically. It additionally helps in recognizing the similitudes and contrasts from each other. Lattice and distributed computing seems, by all accounts, to be a promising model particularly concentrating on institutionalizing APIs, security, interoperability, new plans of action, and element estimating frameworks for complex administrations

MayankaKatyal and Atul Mishra Et al. [8] creators talked about on one hand, static load adjusting plan give simplest reenactment and checking of environment however neglect to demonstrate heterogeneous nature of cloud. Then again, dynamic load adjusting calculation are hard to recreate yet are most appropriate in heterogeneous environment of distributed computing Unlike brought together calculation, conveyed nature of calculation gives better adaptation to non-critical failure however requires higher level of replication and then again, various leveled calculation isolate the heap at various levels of chain of command with upper level hubs asking for administrations of lower level hubs in adjusted way.

S. Subashini N and V. Kavitha Et al. [9] They examine in research, inquiries will focus on application and information security over the cloud, and I expect to build up a structure by which the security philosophy differs powerfully starting with one exchange/correspondence then onto the next. One of the bits of the system may be centered around giving information security by putting away and getting to information in view of meta-information data. This examination depends on the conceptualization of the cloud security in view of genuine security framework where in security relies on upon the necessity and resource estimation of an individual or association.

Saleh Basalamah, Abdulrahman A. Almutairi, Muhammad I. Sarfraz, Walid G. Aref and ArifGhafoor Et al. [10] They portray a novel dispersed design joins standards from security administration and programming building to address distributed computing's security challenges. They display in this article speaks to an exact yet thorough approval outline for get to administration. Utilizing a XML-based assertion of the get to control arrangement for this engineering is a stage toward its usage. These incorporate plans of a validation system, cryptography and key administration, intervention for struggle determination of heterogeneous strategies, programming outline for virtualized assets, coordinating data stream confirmation devices to guarantee non-impedance, and building decisions for SLAs.
Dimitrios Zissis and DimitriosLekkas Et al. [11] They examine, Cloud processing offers sending engineering, with the capacity to address vulnerabilities perceived in conventional IS yet its dynamic qualities can prevent the viability of customary countermeasures. A mix of PKI, LDAP and SSO can address the vast majority of the recognized dangers in distributed computing managing the respectability, privacy, credibility and accessibility of information and interchanges. The arrangement, shows an even level of administration, accessible to all embroiled substances, that understands a security work through alliances, inside which basic trust is kept up.

Jianxin Li, Bo Li, Tianyu Wo, Chunming Hu, JinpengHuai, Lu Liu and K.P. Lam Et al. [12] creators examined, CyberGuarder gives three various types of administrations; to be specific, a virtual machine security benefit, a virtual system security benefit and an approach based trust administration benefit. A few systems concerning the arrangement of honesty check, multi-level NetApp detachment, virtual security apparatus and NetApp asset trust administration administrations have been talked about in some detail. Preparatory outcomes got on their iVIC stage are promising. At present, they are fusing these outcomes and their involvement with Cyber-Guarder in the execute improvement of iVIC, a virtualization foundation for a trial course conveyance framework for both students and postgraduates in Beihang University.

\section{PROBLEM FORMULATION}

In a disseminated framework, dynamic load adjusting should be possible in two distinctive ways: dispersed and noncirculated. In the dispersed one, the dynamic load adjusting calculation is executed by all hubs display in the framework and the assignment of load adjusting is shared among them. The collaboration among hubs to accomplish stack adjusting can take two structures: agreeable and non-helpful [4]. In the first, the hubs work one next to the other to accomplish a typical goal, for instance, to enhance the general reaction time, and so forth. In the second frame, every hub works freely toward an objective neighborhood to it, for instance, to enhance the reaction time of a nearby undertaking. Dynamic load adjusting calculations of conveyed nature, for the most part create a greater number of messages than the nondispersed ones because, each of the hubs in the framework needs to associate with each other hub. An advantage, of this is regardless of the possibility that at least one hubs in the framework fall flat, it won't bring about the aggregate load adjusting procedure to end, it rather would influence the framework execution to some degree. Conveyed dynamic load balancing can present massive weight on a framework in which every hub needs to exchange status data with each other hub in the framework. It is more invaluable when a large portion of the hubs demonstration independently with not very many cooperation's with others. The procedure of load adjusting and assignment booking give a noteworthy part in achievement of distributed computing. In survey handle we found that different component impact the execution of cloud computational in worries of capacity of system information and sharing of asset. The sharing of cloud asset creates a system over-burden, the system over-burden emerges the issue of data transfer capacity and stack flood. Some issue identified with distributed computing is given beneath.

1. Increasing the time traverse for process $[2,1]$

2. Failure of asset allocation $[2,4]$

3. Traffic overhead of network $[2,3]$

4. Waste of resource [11] 
5. Cost of observing of resource [12]

6. Process criticism system [14]

\section{CONCLUSION AND FUTURE SCOPE}

In this paper present the review of load balancing technique in terms of data centers and task deployments. Data centers is important assets of cloud computing, the physical host of data centers is overload the performance and efficiency of cloud environments is automatically degraded. The balancing technique balance the load on physical host of data centers and enhanced the performance of data centers. In the process of review find that various authors used clustering and machine learning technique. the clustering and machine learning technique gives effective balancing technique and reduces the overloaded scenario of cloud enjoyments. The various researcher and scientists used dynamic and static load balancing technique. in dynamic load balancing technique used heuristic function such as ACO, PSO, Genetic algorithm and many more guided searching algorithms. the shared virtual machine allocated the job in dedicated time for the execution of process. In future used classification and prototype model for load balancing.

\section{REFERENCES}

[1] Jia Zhao, Kun Yang, Xiaohui Wei, Yan Ding, Liang Hu, and Gaochao Xu "A Heuristic Clustering-Based Task Deployment Approach for Load Balancing Using Bayes Theorem in Cloud Environment", IEEE, 2016, Pp 305316.

[2] Ariharan V and Sheeja S Manakattu "Neighbour Aware Random Sampling (NARS) algorithm for load balancing in Cloud computing", IEEE, 2015, Pp 1-5.

[3] Catalin-Constantin Usurelu, Mihaela-Catalina Nita, Roxana Istrate, Florin Pop and Nicolae Tapus "Spider Mesh Overlay for Task Load Balancing in Cloud Computing", IEEE, 2015, Pp 433-440.
[4] Mohamed Belkhouraf, Ali Kartit, Hassan Ouahmane, Hamza Kamal Idrissi, Zaid Kartit and Mohamed El Marraki "A secured load balancing architecture for cloud computing based on multiple clusters", IEEE, 2015, Pp $1-6$.

[5] Yi Wei and M. Brian Blake "Service-Oriented Computing and Cloud Computing", IEEE, 2011, Pp 72-75.

[6] Anurag Jain and Rajneesh Kumar "Scalable and Trustworthy Load Balancing Technique for Cloud Environment", International Journal of Engineering and Technology, 2016, Pp 1245-1251.

[7] Naidila Sadashiv and S. M Dilip Kumar "Cluster, Grid and Cloud Computing: A Detailed Comparison", IEEE, 2011, Pp 477-482.

[8] MayankaKatyal and Atul Mishra "A Comparative Study of Load Balancing Algorithms in Cloud Computing Environment", International Journal of Distributed and Cloud Computing, 2013, Pp 5-14.

[9] S. Subashini N and V. Kavitha "A survey on security issues in service delivery models of cloud computing", Journal of Network and Computer Applications, 2010, Pp 1-11.

[10] Saleh Basalamah, Abdulrahman A. Almutairi, Muhammad I. Sarfraz, Walid G. Aref and ArifGhafoor "A Distributed Access Control Architecture for Cloud Computing”, IEEE, 2012, Pp 36-44.

[11] Dimitrios Zissis and DimitriosLekkas “Addressing cloud computing security issues", Future Generation Computer Systems, 2012, Pp 583-592.

[12] Jianxin Li, Bo Li, Tianyu Wo, Chunming $\mathrm{Hu}$, JinpengHuai, Lu Liu and K.P. Lam "CyberGuarder: A virtualization security assurance architecture for green cloud computing" Future Generation Computer Systems, 2012, Pp 379-390. 